(c) 2019 IEEE. Personal use of this material is permitted. Permission from IEEE must be obtained for all other uses, in any current or future media, including reprinting/republishing this material for advertising or promotional purposes, creating new collective works, for resale or redistribution to servers or lists, or reuse of any copyrighted component of this work in other work.

\title{
A generalization of the Choquet integral defined in terms of the Möbius transform
}

\author{
Javier Fernandez, Humberto Bustince, Senior Member, IEEE, Lubomíra Horanská, Radko Mesiar \\ and Andrea Stupňanová
}

\begin{abstract}
In this work we propose a generalization of the Choquet integral starting from its definition in terms of the Möbius transform. We modify the product on $\mathbb{R}$ considered in the Lovász extension form of the Choquet integral into a function $F$, and we discuss the properties of this new functional. For a fixed $n$, a complete description of all $F$ yielding an $n$-ary aggregation function with a fixed diagonal section, independently of the considered fuzzy measure, is given, and several particular examples are presented. Finally, all functions $F$ yielding an aggregation function, independently of the number $n$ of inputs and of the considered fuzzy measure, are characterized, and related aggregation functions are shown to be just the Choquet integrals over the distorted inputs.
\end{abstract}

Index Terms-Aggregation function, Fuzzy measure, Choquet integral, Möbius transform.

\section{INTRODUCTION}

In recent years there has been a growing interest in the developing of generalized forms of the Choquet integral, due to its flexibility in many different applications. Most of these generalizations are based on considering the usual expression of the Choquet integral in terms of a sum of products, and replacing the product by more general functions. The resulting operators are quite different from the usual Choquet integral (for instance, they do not need to be aggregation functions any more) but have provided good results in different applications.

In this sense, it seems logical to explore further this approach by considering other possible expressions of the Choquet integral. The objective of this work is to propose a new family of fuzzy measure-based aggregation function, arising from the expression of the Choquet integral in terms of the Möbius transform of the fuzzy measure which it is based on. As this new class of functions covers the class of

J. Fernandez and H. Bustince are with Department of Statistics, Computer Science and Mathematics and Institute of Smart Cities, Universidad Pública de Navarra, Campus Arrosadía s/n, P.O. Box 31006, Pamplona, Spain, (e-mail: \{bustince,fcojavier.fernandez\}@unavarra.es).

L. Horanská is with Institute of Information Engineering, Automation and Mathematics, Faculty of Chemical and Food Technology, Slovak University of Technology in Bratislava, Radlinského 9, 81237 Bratislava 1, Slovak Republic, (e-mail: lubomira.horanska@stuba.sk).

R. Mesiar is with the Department of Mathematics and Descriptive Geometry, Faculty of Civil Engineering, Slovak University of Technology in Bratislava, Radlinského 11, 81005 Bratislava 1, Slovak Republic, and also with the Department of Algebra and Geometry, Faculty of Science, Palacký University Olomouc, 17. listopadu 12, 77146 Olomouc, Czech Republic (email: radko.mesiar@stuba.sk).

A. Stupňanová is with the Department of Mathematics and Descriptive Geometry, Faculty of Civil Engineering, Slovak University of Technology in Bratislava, Radlinského 11, 81005 Bratislava 1, Slovak Republic (e-mail: andrea.stupnanova@stuba.sk).

Manuscript received January 4, 2019. standard Choquet integrals, it is reasonable to expect that the behaviour of these functions on those problems where Choquet integrals work well will also be good. In fact, we intend to develop an analysis of some applications in our future works. In particular, as the Möbius transform of a given fuzzy measure may include negative values, this generalization is appropriate to be used in those cases where the use of negative values may be considered as natural, as it is the case, for instance, of neural networks (where negative weights are relevant to represent inhibitory behaviour).

The structure of this paper is as follows. In the next section, some necessary preliminaries are given. Section 3 brings our main results and it deals with a generalization of the Choquet integral based on the Möbius transform of fuzzy measures. Section 4 contains results concerning the function $F$ generalizing the standard product and yielding an aggregation function with a fixed diagonal section, independently of the considered fuzzy measure. Finally, some concluding remarks are given.

\section{Preliminaries}

In what follows, we denote $N=\{1, \ldots, n\}$ for a positive integer $n$.

Definition 2.1 ([1]): A function $H:[0,1]^{n} \rightarrow[0,1]$ is said to be an aggregation function whenever the following conditions hold:

(A1) $H$ is increasing ${ }^{1}$ in each argument: for each $i \in$ $N$, if $x_{i} \leq y$, then $H\left(x_{1}, \ldots, x_{i}, \ldots, x_{n}\right) \leq$ $H\left(x_{1}, \ldots, x_{i-1}, y, x_{i+1}, \ldots, x_{n}\right)$;

(A2) $H$ satisfies the boundary conditions: $H(0, \ldots, 0)=$ 0 and $H(1, \ldots, 1)=1$.

Formally, unary aggregation functions are just functions $H:[0,1] \rightarrow[0,1]$ which are increasing and satisfy $H(0)=$ $0, H(1)=1$. The class of all $n$-ary aggregations functions will be denoted by $\mathcal{A}_{(n)}$.

The concept of fuzzy measure [2], [3] is important for defining the Choquet integral. Fuzzy measures capture the relationship among the aggregated elements.

Definition 2.2: A function $\mu: 2^{N} \rightarrow[0,1]$ is said to be a fuzzy measure, if the following conditions hold:

(M1) Increasingness: if $A \subseteq B \subseteq N$, then $\mu(A) \leq \mu(B)$;

(M2) Boundary conditions: $\mu(\emptyset)=0$ and $\mu(N)=1$.

The class of all fuzzy measures on $2^{N}$ will be denoted by $\mathfrak{M}_{(n)}$.

\footnotetext{
${ }^{1}$ By an increasing (decreasing) function we do not mean a strictly increasing (decreasing) function.
} 
The Choquet integral generalizes the Lebesgue integral, which is defined considering additive measures. The Choquet integral, however, considers fuzzy measures. The discrete Choquet integral [2] is defined on finite spaces as follows.

Definition 2.3 ([1], [4]): Let $\mu: 2^{N} \rightarrow[0,1]$ be a fuzzy measure. The discrete Choquet integral is the function $\mathbf{C h}_{\mu}:[0,1]^{n} \rightarrow[0,1]$, defined, for all $\mathbf{x}=\left(x_{1}, \ldots, x_{n}\right) \in$ $[0,1]^{n}$, by:

$$
\mathbf{C h}_{\mu}(\mathbf{x})=\sum_{i=1}^{n}\left(x_{(i)}-x_{(i-1)}\right) \cdot \mu\left(A_{(i)}\right),
$$

where $\left(x_{(1)}, \ldots, x_{(n)}\right)$ is an increasing permutation on the input $\mathrm{n}$-tuple $\mathrm{x}$, i.e., $0 \leq x_{(1)} \leq \ldots \leq x_{(n)}$, where $x_{(0)}=0$ and $A_{(i)}=\{(i), \ldots,(n)\}$ is the subset of indices corresponding to the $n-i+1$ largest components of $\mathbf{x}$.

Formula (1) was generalized in several recent works replacing the product by some appropriate bivariate function $F$, see [5]. As another example, considering a copula $C, C C$-integrals were introduced in [6]. Observe that if $C=$ Min, then the Sugeno integral [7] is recovered.

The Choquet integral is an idempotent aggregation function, i.e., $\mathbf{C h}_{\mu}(x, \ldots, x)=x$ for all $x \in[0,1]$. A concept closely related to the notion of Choquet integral is that of Möbius transform, that we recall now and which is at the core of the present work.

Definition 2.4: Let $\mu: 2^{N} \rightarrow[0,1]$ be a fuzzy measure. The Möbius transform of $\mu$ is a function $\mathcal{M}: 2^{N} \rightarrow \mathbb{R}$ given by

$$
\mathcal{M}(A)=\sum_{B \subseteq A}(-1)^{|A \backslash B|} \mu(B)
$$

for every $A \subseteq N$, where $|A|$ denotes the cardinality of the set A.

Möbius transform provides a different representation for a fuzzy measure $\mu$. Furthermore, it is invertible by means of the so-called Zeta transform, that we recall now.

Proposition 2.1: Let $\mu: 2^{N} \rightarrow[0,1]$ be a fuzzy measure and let $\mathcal{M}$ be its Möbius transform. Then, it holds

$$
\mu(A)=\sum_{B \subseteq A} \mathcal{M}(B)
$$

for every $A \subseteq N$.

Taking into account Proposition 2.1, the Choquet integral can alternatively be written as follows.

Proposition 2.2: Let $\mu: 2^{N} \rightarrow[0,1]$ be a fuzzy measure. Then it holds

$$
\mathbf{C h}_{\mu}(\mathbf{x})=\sum_{\emptyset \neq B \subseteq N} \mathcal{M}(B) \min _{i \in B}\left\{x_{i}\right\}
$$

for any $\mathbf{x}=\left(x_{1}, \ldots, x_{n}\right) \in[0,1]^{n}$.

Remark 2.3: Note that for the Möbius transform $\mathcal{M}$ of any fuzzy measure $\mu \in \mathfrak{M}_{(n)}$ it holds that $\mathcal{M}(\emptyset)=0$, so we are not loosing any relevant information by omitting this summand in (3).

Remark 2.4: Formula (3) is known from the game theory as the Lovász extension [8]. Indeed, the aggregation function $\mathrm{Ch}_{\mu}$ monotonically extends the fuzzy measure $\mu$ in the sense that $\mathbf{C h}_{\mu}\left(\mathbf{1}_{A}\right)=\mu(A)$, for any set $A \subseteq N$, where $\mathbf{1}_{A}: N \rightarrow$ $\{0,1\}$ is the characteristic function of $A$, i.e., $\left(\mathbf{1}_{A}\right)(i)=1$ if $i \in A$ and $\left(\mathbf{1}_{A}\right)(i)=0$ otherwise.

\section{A Generalization OF THE ChOQUet INTEgRAL EXPRESSED IN TERMS OF THE MÖBIUS TRANSFORM}

In this section, we consider a possible generalization of the formula (3) replacing the product by more general functions, similarly as it was done with the expression (1) in [9], [5]. To obtain this generalization, we replace the product by more general functions with some kind of regularity. In particular, we propose the following definition.

Definition 3.1: Let $n \geq 1, \mu \in \mathfrak{M}_{(n)}$ and $\mathcal{M}$ be the Möbius transform of $\mu$. Let $F: \mathbb{R} \times[0,1] \rightarrow \mathbb{R}$ be a function bounded on $[0,1]^{2}$. We define the function $\mathfrak{I}_{\mu}^{F}:[0,1]^{n} \rightarrow \mathbb{R}$ as

$$
\mathfrak{I}_{\mu}^{F}(\mathbf{x})=\sum_{\emptyset \neq B \subseteq N} F\left(\mathcal{M}(B), \min _{i \in B}\left\{x_{i}\right\}\right) .
$$

Example 3.1: Taking $F(u, v)=u v$ we recover the standard Choquet integral.

Example 3.2: Let $f:[0,1] \rightarrow[0,1]$ be an increasing function. Taking $F(u, v)=u f(v)$ we get:

$$
\mathfrak{I}_{\mu}^{F}(\mathbf{x})=\mathbf{C h}_{\mu}\left(f\left(x_{1}\right), \ldots, f\left(x_{n}\right)\right) .
$$

Note that $\mathfrak{I}_{\mu}^{F}$ is an $n$-ary aggregation function if and only if $f$ is a unary aggregation function.

In general, taking two different functions $F_{1}, F_{2}$ and a fuzzy measure $\mu \in \mathfrak{M}_{(n)}$, the corresponding functions $\mathfrak{I}_{\mu}^{F_{1}}, \mathfrak{I}_{\mu}^{F_{2}}$ need not necessarily be different, as the following proposition illustrates.

Proposition 3.1: Let $F: \mathbb{R} \times[0,1] \rightarrow \mathbb{R}$ be a function bounded on $[0,1]^{2}$ and $c \in \mathbb{R}$. Let $F_{c}: \mathbb{R} \times[0,1] \rightarrow \mathbb{R}$ be a function defined by

$$
F_{c}(x, y)=F(x, y)+c\left(x-\frac{1}{2^{n}-1}\right) .
$$

Then, for any $\mu \in \mathfrak{M}_{(n)}$, it holds

$$
\mathfrak{I}_{\mu}^{F}(\mathbf{x})=\mathfrak{I}_{\mu}^{F_{c}}(\mathbf{x})
$$

for all $\mathbf{x} \in[0,1]^{n}$.

Proof: Since $\sum_{\emptyset \neq B \subseteq N} c\left(\mathcal{M}(B)-\frac{1}{2^{n}-1}\right)=0$, the result follows.

Remark 3.2: Due to the Proposition 3.1, one can consider $F(0,0)=0$ with no loss of generality.

As it can easily be seen, considering a general function $F: \mathbb{R} \times[0,1] \rightarrow \mathbb{R}, \Im_{\mu}^{F}$ need not be an aggregation function. We are interested in just those functions $F$ yielding an aggregation function $\mathfrak{I}_{\mu}^{F}$ for all fuzzy measures $\mu \in \mathfrak{M}_{(n)}$.

Definition 3.2: Let $n \geq 1$. The class $\mathcal{F}_{n}$ is defined as

$$
\begin{array}{r}
\mathcal{F}_{n}=\left\{F: \mathbb{R} \times[0,1] \rightarrow \mathbb{R} \mid \mathfrak{I}_{\mu}^{F} \in \mathcal{A}_{(n)}\right. \\
\text { for any } \left.\mu \in \mathfrak{M}_{(n)}\right\} .
\end{array}
$$

The class $\mathcal{F}$ is defined as

$$
\mathcal{F}=\bigcap_{n \geq 1} \mathcal{F}_{n} .
$$

Clearly, the class $\mathcal{F}$ is not empty, since the function $F(u, v)=$ $u v$ belongs there. Note that in Def. 3.1 we have required $F$ to be bounded on $[0,1]^{2}$. If $F$ is not bounded, then we can 
find a fuzzy measure $\mu$ such that $\mathfrak{I}_{\mu}^{F}$ does not belong to the corresponding class $\mathcal{A}_{(n)}$.

Remark 3.3: Take $n=1$. There exists only one fuzzy measure $\mu \in \mathfrak{M}_{(1)}$ given by $\mu(\emptyset)=0$ and $\mu(\{1\})=1$. So in this case, we have

$$
\mathfrak{I}_{\mu}^{F}(x)=F(1, x)
$$

for every $x \in[0,1]$. This means that

$$
\begin{aligned}
& \mathcal{F}_{1}=\{F: \mathbb{R} \times[0,1] \rightarrow \mathbb{R} \mid F(1,0)=0, F(1,1)=1, \\
& F(1, x) \text { is increasing w.r.t. } x\} \text {. }
\end{aligned}
$$

Note that $F \in \mathcal{F}_{1}$ if and only if $F(1,$.$) is an unary aggregation$ function. Obviously, for $F \in \mathcal{F}_{1}$ the values of $F(u, v)$ for $u \neq 1$ are irrelevant, since the only non-empty subset of $\{1\}$ is just $\{1\}$, and $\mu(\{1\})=\mathcal{M}(\{1\})=1$.

Proposition 3.4: For any $n \geq 1$, the class $\mathcal{F}_{n}$ is convex. Proof: Take $F_{1}, F_{2} \in \mathcal{F}_{n}$ and $\lambda \in[0,1]$. Let $F_{0}=\lambda F_{1}+(1-$ $\lambda) F_{2}$ be a convex combination of $F_{1}$ and $F_{2}$ and $\mu \in \mathfrak{M}_{(n)}$. Then

$$
\mathfrak{I}_{\mu}^{F_{0}}(\mathbf{x})=\lambda \mathfrak{I}_{\mu}^{F_{1}}(\mathbf{x})+(1-\lambda) \mathfrak{I}_{\mu}^{F_{2}}(\mathbf{x})
$$

Since $F_{1}, F_{2} \in \mathcal{F}_{n}$, both $\mathfrak{I}_{\mu}^{F_{1}}$ and $\mathfrak{I}_{\mu}^{F_{2}}$ are aggregation functions. Taking into account that a convex combination of aggregation functions is an aggregation function, the result follows.

Since the intersection of convex classes is convex, we have the following observation.

Corollary 3.5: The class $\mathcal{F}$ is convex.

Example 3.3: Given $n \geq 1$, consider the function $F_{n}: \mathbb{R} \times$ $[0,1] \rightarrow \mathbb{R}$ given by

$$
F_{n}(u, v)=\frac{v}{2^{n}-1} .
$$

Then, for any fuzzy measure $\mu \in \mathfrak{M}_{(n)}$ we obtain

$$
\begin{aligned}
& \mathfrak{I}_{\mu}^{F_{n}}(\mathbf{x}) \\
& =\frac{1}{2^{n}-1}\left(2^{n-1} x_{\sigma(1)}+2^{n-2} x_{\sigma(2)}+\cdots+2 x_{\sigma(n-1)}+x_{\sigma(n)}\right),
\end{aligned}
$$

and

which is independent of $\mu$, thus we can write $\mathfrak{I}_{\mu}^{F_{n}}=\mathfrak{I}^{F_{n}}$. Note that $\mathfrak{I}^{F_{n}}$ is a particular OWA operator, and hence it is an aggregation function, so, for any $n \geq 1, F_{n} \in \mathcal{F}_{n}$.

Clearly, if $n \neq m$, then $F_{n} \notin \mathcal{F}_{m}$. Hence, for any $n \geq 2$, $F_{n} \notin \mathcal{F}$. Observe that $\mathfrak{I}^{F_{n}}=\mathbf{C h}_{\mu_{n}}$, where $\mu_{n}: 2^{N} \rightarrow[0,1]$ is a symmetric fuzzy measure given by

$$
\mu_{n}(E)=\frac{2^{|E|}-1}{2^{n}-1}, E \subseteq N
$$

Example 3.4: According to Corollary 3.5, for any $\lambda \in[0,1]$ also the function $G_{n}: \mathbb{R} \times[0,1] \rightarrow \mathbb{R}$ given by

$$
G_{n}(u, v)=\lambda u v+\frac{(1-\lambda) v}{2^{n}-1}
$$

belongs to $\mathcal{F}_{n}$ for any $n \geq 2$ and we have

$$
\mathfrak{I}_{\mu}^{G_{n}}=\lambda \mathbf{C h}_{\mu}+(1-\lambda) \mathfrak{I}^{F_{n}} .
$$

If $\mu$ is a symmetric fuzzy measure, then $\mathfrak{I}_{\mu}^{G_{n}}$ is an OWA operator.

\section{IV. $\mathfrak{I}_{\mu}^{F}$ WITH A FIXED DIAGONAL SECTION INDEPENDENT OF FUZZY MEASURE}

It turns out that a complete characterization of the class $\mathcal{F}_{n}$ for $n \geq 2$ is a difficult problem. However, in this section we give a characterization of the subclass of $\mathcal{F}_{n}$ consisting of all functions $F$ yielding $\mathfrak{I}_{\mu}^{F}$ with the same diagonal section for all fuzzy measures $\mu \in \mathfrak{M}_{(n)}$.

Recall that for any $n \in \mathbb{N}$ and $n$-ary aggregation function $H:[0,1]^{n} \rightarrow[0,1]$, the related diagonal section $\delta_{H}:[0,1] \rightarrow$ $[0,1]$ given by

$$
\delta_{H}(x)=H(x, \ldots, x)
$$

is a unary aggregation function, i.e., $\delta \in \mathcal{A}_{(1)}$. Vice versa, for any $\delta \in \mathcal{A}_{(1)}$ and $n \in \mathbb{N}$, there is an $n$-ary aggregation function $H$ so that $\delta=\delta_{H}$. As a typical example, one can consider $H\left(x_{1}, \ldots, x_{n}\right)=\frac{1}{n} \sum_{i=1}^{n} \delta\left(x_{i}\right)$. Similarly, for any fuzzy measure $\mu \in \mathfrak{M}_{(n)}, H$ given by $H\left(x_{1}, \ldots, x_{n}\right)=$ $\mathbf{C h}_{\mu}\left(\delta\left(x_{1}\right), \ldots, \delta\left(x_{n}\right)\right)$ satisfies $\delta_{H}=\delta$ as $\mathbf{C h}_{\mu}$ is an idempotent aggregation function. As another example, modifying Example 3.3, for $n \in \mathbb{N}$ one can consider $H:[0,1]^{n} \rightarrow[0,1]$ given, for any fuzzy measure $\mu \in \mathfrak{M}_{(n)}$, as

$$
H(\mathbf{x})=\mathfrak{I}_{\mu}^{F_{n, \delta}}(\mathbf{x}),
$$

where $F_{n, \delta}(u, v)=\frac{\delta(v)}{2^{n}-1}$. Obviously, $\delta_{H}=\delta$, and $F_{n, \delta} \in \mathcal{F}_{n}$.

Definition 4.1: Let $n \geq 1$ and $\delta \in \mathcal{A}_{(1)}$. The class $F_{n, \delta}$ is defined as

$$
\begin{array}{r}
\mathcal{F}_{n, \delta}=\left\{F: \mathbb{R} \times[0,1] \rightarrow \mathbb{R} \mid \mathfrak{I}_{\mu}^{F} \in \mathcal{A}_{(n)}, \delta_{\mathfrak{I}_{\mu}^{F}}=\delta\right. \\
\text { for any } \left.\mu \in \mathfrak{M}_{(n)}\right\} .
\end{array}
$$

The class $\mathcal{F}_{\delta}$ is defined as

$$
\mathcal{F}_{\delta}=\bigcap_{n \geq 1} \mathcal{F}_{n, \delta} .
$$

Observe that $\mathcal{F}_{n, \delta} \subset \mathcal{F}_{n}$ for any $\delta \in \mathcal{A}_{(1)}$ and $n \in \mathbb{N}$, so $\mathcal{F}_{\delta} \subset \mathcal{F}$. Moreover,

$$
\begin{gathered}
\mathcal{F}_{1, \delta}=\{F \mid F(1, v)=\delta(v)\} \\
\bigcup_{\delta \in \mathcal{A}_{(1)}} \mathcal{F}_{1, \delta}=\mathcal{F}_{1} .
\end{gathered}
$$

We will start with characterization of the class $\mathcal{F}_{n \text {,id }}$ of all functions $F$ generating idempotent aggregation functions $\mathfrak{I}_{\mu}^{F}$ for all $\mu \in \mathfrak{M}_{(n)}$, i.e.,

$$
\begin{array}{r}
\mathcal{F}_{n, \text { id }}=\left\{F: \mathbb{R} \times[0,1] \rightarrow \mathbb{R} \mid \mathfrak{I}_{\mu}^{F} \in \mathcal{A}_{(n)}, \delta_{\mathfrak{I}_{\mu}^{F}}=\mathrm{id}\right. \\
\text { for any } \left.\mu \in \mathfrak{M}_{(n)}\right\} .
\end{array}
$$

Denote $\mathcal{R}_{n}$ the range of Möbius transforms of all possible fuzzy measures on $N=\{1, \ldots, n\}$. Recall that $\mathcal{R}_{2}=$ $[-1,1], \mathcal{R}_{3}=[-2,1]$, etc.

Theorem 4.1: Let $n \geq 2$. Then the following are equivalent.

(i) $F \in \mathcal{F}_{n \text {,id. }}$

(ii) There is a function $h:[0,1] \rightarrow \mathbb{R}$, such that, for any $(x, y) \in[0,1]^{2}$, with $x<y$, it holds

$$
-\frac{y-x}{2^{n}-2} \leq h(y)-h(x) \leq y-x,
$$

and, for all $(u, v) \in \mathcal{R}_{n} \times[0,1]$, it holds 


$$
F(u, v)=u h(v)+\frac{v-h(v)}{2^{n}-1} .
$$

Proof:

(i) $\Rightarrow($ ii $)$ : Let $F \in \mathcal{F}_{n \text {,id }}$ and $\mu \in \mathfrak{M}_{(n)}$ be the fuzzy measure such that $\mu(N)=1, \mu(N \backslash\{n\})=a$ and $\mu(N \backslash\{n-1\})=b$ for some $a, b \in[0,1]$ and vanishing otherwise. Due to the idempotency of $\mathfrak{I}_{\mu}^{F}$, we have

$$
\begin{aligned}
x & =\mathfrak{I}_{\mu}^{F}(x, \ldots, x) \\
& =F(a, x)+F(b, x)+F(1-a-b, x)+\left(2^{n}-4\right) F(0, x),
\end{aligned}
$$

for all $x \in[0,1]$.

For a fixed $x \in[0,1]$, denote $F(u, x)=f(u), u \in[-1,1]$. Then $f:[-1,1] \rightarrow \mathbb{R}$ satisfies

$$
f(a)+f(b)+f(1-a-b)+\left(2^{n}-4\right) f(0)=x,
$$

for any $a, b \in[0,1]$. Thus, for any $a, b \in[0,1]$ we have

$$
\begin{array}{r}
f(a)+f(1-a)+\left(2^{n}-3\right) f(0)=x, \\
f(a)+f(1-b)+f(b-a)+\left(2^{n}-4\right) f(0)=x,
\end{array}
$$

hence,

$$
f(1-a)+f(0)=f(1-b)+f(b-a) .
$$

Denote $s=1-a, t=1-b$. Clearly, $s, t \in[0,1]$ and

$$
f(s)+f(0)=f(t)+f(s-t) .
$$

Let $g=f-f(0)$. Then (8) can be rewritten as

$$
g(s)=g(t)+g(s-t),
$$

for any $s, t \in[0,1]$. Obviously, if $s=t=0$, we have $g(0)=$ $2 g(0)$, i.e., $g(0)=0$. Similarly, taking $s=0$, we get $0=$ $g(t)+g(-t)$, i.e., $g$ is an odd function. Considering $s \geq t$, $s-t=u$, we obtain the Cauchy equation $g(t+u)=g(t)+$ $g(u)$ for all $t, u \in[0,1]$ such that $u+t \in[0,1]$. Due to the boundedness of $F$ on $[0,1]^{2}$, also $g$ is bounded, therefore $g(t)=c \cdot t$ for some constant $c$ (for the proof see, e.g., [10]). Consequently,

$$
f(t)=c \cdot t+f(0) .
$$

For $a=b=\frac{1}{3}$, formulae (7) and (10) give

$$
x=3 \cdot f\left(\frac{1}{3}\right)+\left(2^{n}-4\right) f(0)=c+\left(2^{n}-1\right) f(0),
$$

thus $f(0)=\frac{x-c}{2^{n}-1}$. Finally, denoting $c=h(x)$, we obtain

$$
F(u, x)=f(u)=u \cdot h(x)+\frac{x-h(x)}{2^{n}-1},
$$

for any $x \in[0,1]$ and $u \in[-1,1]=\mathcal{R}_{2}$. We will show the validity of (6) for all $u \in \mathcal{R}_{n}$ by induction. Suppose that formula (6) holds for all $u \in \mathcal{R}_{n-1}$. Observe that for any fuzzy measure $\mu \in \mathfrak{M}_{(n)}$ and $A \subsetneq N$ it holds $\mathcal{M}(A) \in \mathcal{R}_{n-1}$. Then, for any $u \in \mathcal{R}_{n} \backslash \mathcal{R}_{n-1}$, there is a fuzzy measure $\widetilde{\mu} \in \mathfrak{M}_{(n)}$ such that for the corresponding Möbius transform $\mathcal{M}(N)=u$. Hence,

$$
\begin{aligned}
x & =\mathfrak{I}_{\widetilde{\mu}}^{F}(x, \ldots, x) \\
& =F(u, x)+\sum_{\emptyset \neq A \subsetneq N}\left(\mathcal{M}(A) h(x)+\frac{x-h(x)}{2^{n}-1}\right) \\
& =F(u, x)+(1-u) h(x)+\left(2^{n}-2\right) \frac{x-h(x)}{2^{n}-1},
\end{aligned}
$$

which implies

for all $u \in \mathcal{R}_{n}$.

For proving conditions given by (5), consider $\left(x_{1}, \ldots, x_{n}\right) \in[0,1]^{n}$ such that $x_{1}<x_{2}<\cdots<x_{n}$. Due to the monotonicity of $\mathfrak{I}_{\mu}^{F}$ it holds

$$
\mathfrak{I}_{\mu}^{F}\left(x_{2}, x_{2}, x_{3}, \ldots, x_{n}\right) \geq \mathfrak{I}_{\mu}^{F}\left(x_{1}, x_{2}, x_{3}, \ldots, x_{n}\right),
$$

and hence, we obtain

$$
\sum_{1 \in A} F\left(\mathcal{M}(A), x_{2}\right) \geq \sum_{1 \in A} F\left(\mathcal{M}(A), x_{1}\right) .
$$

Using (6) we get

$$
\begin{aligned}
& h\left(x_{2}\right) \sum_{1 \in A} \mathcal{M}(A)+2^{n-1} \frac{x_{2}-h\left(x_{2}\right)}{2^{n}-1} \\
& \geq \quad h\left(x_{1}\right) \sum_{1 \in A} \mathcal{M}(A)+2^{n-1} \frac{x_{1}-h\left(x_{1}\right)}{2^{n}-1} .
\end{aligned}
$$

Taking the maximal fuzzy measure $\mu^{*}$ defining by $\mu^{*}(A)=1$ for all $\emptyset \neq A \subseteq N$ and $\mu^{*}(\emptyset)=0$ we have $\sum_{1 \in A} \mathcal{M}(A)=0$ and therefore

$$
h\left(x_{2}\right)-h\left(x_{1}\right) \leq x_{2}-x_{1} .
$$

Similarly, whence

$$
\mathfrak{I}_{\mu}^{F}\left(x_{1}, \ldots, x_{n-1}, x_{n}\right) \geq \mathfrak{I}_{\mu}^{F}\left(x_{1}, \ldots x_{n-1}, x_{n-1}\right),
$$

we have

$$
F\left(\mathcal{M}(\{n\}), x_{n}\right) \geq F\left(\mathcal{M}(\{n\}), x_{n-1}\right) .
$$

Thus, using (6)

$$
\begin{aligned}
& \mathcal{M}(\{n\}) h\left(x_{n}\right)+\frac{x_{n}-h\left(x_{n}\right)}{2^{n}-1} \\
\geq & \mathcal{M}(\{n\}) h\left(x_{n-1}\right)+\frac{x_{n-1}-h\left(x_{n-1}\right)}{2^{n}-1} .
\end{aligned}
$$

Taking $\mu \in \mathfrak{M}_{(n)}$ such that $\mu(\{n\})=\mathcal{M}(\{n\})=1$, we get

$$
-\frac{x_{n}-x_{n-1}}{2^{n}-2} \leq h\left(x_{n}\right)-h\left(x_{n-1}\right)
$$

Summarizing, we obtain formula (5).

$(i i) \Rightarrow(i)$ : Applying (6), we see that, for any $\mu \in \mathfrak{M}_{(n)}$, it holds

$\mathfrak{I}_{\mu}^{F}(x, \ldots, x)=h(x) \sum_{\emptyset \neq A \subseteq N} \mathcal{M}(A)+\left(2^{n}-1\right) \cdot \frac{x-h(x)}{2^{n}-1}=x$,

i.e., $\mathfrak{I}_{\mu}^{F}$ is an idempotent function. We will show just the monotonicity of $\mathfrak{I}_{\mu}^{F}$ in the first variable, the monotonicity in 
the other variables can be shown similarly. For the sake of simplicity, consider $\left(x_{1}, \ldots, x_{n}\right) \in[0,1]^{n}$ and $\varepsilon>0$ such that $x_{1}<x_{1}+\varepsilon<x_{2}<\cdots<x_{n}$. Then

$$
\begin{aligned}
& \mathfrak{I}_{\mu}^{F}\left(x_{1}+\varepsilon, x_{2}, \ldots, x_{n}\right)-\mathfrak{I}_{\mu}^{F}\left(x_{1}, x_{2}, \ldots, x_{n}\right) \\
& =\left(h\left(x_{1}+\varepsilon\right)-h\left(x_{1}\right)\right) \sum_{1 \in A \subseteq N} \mathcal{M}(A) \\
& -2^{n-1}\left(\frac{x_{1}+\varepsilon-h\left(x_{1}+\varepsilon\right)}{2^{n}-1}-\frac{x_{1}-h\left(x_{1}\right)}{2^{n}-1}\right) \\
& =\left(h\left(x_{1}+\varepsilon\right)-h\left(x_{1}\right)\right)\left(\sum_{1 \in A \subseteq N} \mathcal{M}(A)-\frac{2^{n-1}}{2^{n}-1}\right)+\frac{2^{n-1}}{2^{n}-1} \varepsilon .
\end{aligned}
$$

Using (5) we have $(h(x+\varepsilon)-h(x)) \in\left[-\frac{1}{2^{n}-2} \varepsilon, \varepsilon\right]$. Moreover, since

$$
\begin{aligned}
\sum_{1 \in A \subseteq N} \mathcal{M}(A) & =\sum_{\emptyset \neq A \subseteq N} \mathcal{M}(A)-\sum_{1 \notin A \subseteq N} \mathcal{M}(A) \\
& =1-\sum_{1 \notin A \subseteq N} \mathcal{M}(A) \\
& \in[0, n-1],
\end{aligned}
$$

we have

$$
\sum_{1 \in A \subseteq N} \mathcal{M}(A)-\frac{2^{n-1}}{2^{n}-1} \in\left[-\frac{2^{n-1}}{2^{n}-1},-\frac{2^{n-1}}{2^{n}-1}+n-1\right]
$$

and thus

$$
\mathfrak{I}_{\mu}^{F}\left(x_{1}+\varepsilon, x_{2}, \ldots, x_{n}\right)-\mathfrak{I}_{\mu}^{F}\left(x_{1}, x_{2}, \ldots, x_{n}\right) \in[0, \varepsilon],
$$

i.e., $\mathfrak{I}_{\mu}^{F}\left(x_{1}+\varepsilon, x_{2}, \ldots, x_{n}\right) \geq \mathfrak{I}_{\mu}^{F}\left(x_{1}, x_{2}, \ldots, x_{n}\right)$. As this claim holds for any $\mu \in \mathfrak{M}_{(n)}$, it follows that $F \in \mathcal{F}_{n \text {,id. Note }}$ that, as a by-product, we have also proved 1-Lipschitzianity of $\mathfrak{I}_{\mu}^{F}$.

Remark 4.2: Note that, requiring the increasingness of $\mathfrak{I}_{\mu}^{F}$ just along the direction defined by one of the vectors of the canonical basis, the proof of Theorem 4.1 remains valid and hence we recover a pre-aggregation function. This can be of interest for a future work.

Remark 4.3: Let $c \in \mathbb{R}, h:[0,1] \rightarrow \mathbb{R}$ be a function fullfiling (5). Taking functions $F$ and $F_{c}$ generated via (6) by $h$ and $h_{c}=h+c$, respectively, we obtain

$$
F_{c}(u, v)=F(u, v)+c\left(u-\frac{1}{2^{n}-1}\right) .
$$

Then, according to Proposition 3.1, we have $\mathfrak{I}_{\mu}^{F_{c}}=\mathfrak{I}_{\mu}^{F}$. It means that, for all $c \in \mathbb{R}$, corresponding functions $h_{c}$ yield the same function $\mathfrak{I}_{\mu}^{F}$. Hence, among all functions $h_{c}$ yielding via (6) the same aggregation function $\mathfrak{I}_{\mu}^{F}$, we can choose a function $h_{c_{0}}$ satisfying $h_{c_{0}}(0)=0$. Note that in this case, the range of $h_{c_{0}}$ is a subset of $\left[-\frac{1}{2^{n}-2}, 1\right]$.

The following two corollaries are easy consequences of Theorem 4.1.

Corollary 4.4: The set $\mathcal{F}_{\text {id }}$ is a singleton, $\mathcal{F}_{\text {id }}=$ $\{F \mid F(u, v)=u v\}$ and then, for any $n \in \mathbb{N}$ and a fuzzy measure $\mu \in \mathfrak{M}_{(n)}, \mathfrak{I}_{\mu}^{F}=\mathbf{C h}_{\mu}$.

Note that the function $h$ considered in Theorem 4.1 need not be increasing, in general.
Corollary 4.5: Let $h:[0,1] \rightarrow[0,1], h(0)=0$ be an increasing 1-Lipschitz function. Then, for any $n \in \mathbb{N}$ and a fuzzy measure $\mu \in \mathfrak{M}_{(n)}, \mathfrak{I}_{\mu}^{F}:[0,1]^{n} \rightarrow[0,1]$ is an idempotent aggregation function given by

$$
\begin{aligned}
& \mathfrak{I}_{\mu}^{F}\left(x_{1}, \ldots, x_{n}\right) \\
& =\mathbf{C h}_{\mu}\left(h\left(x_{1}\right), \ldots, h\left(x_{n}\right)\right)+\frac{1}{2^{n}-1} \sum_{i=1}^{n} 2^{n-i}\left(x_{\sigma(i)}-h\left(x_{\sigma(i)}\right)\right)
\end{aligned}
$$

where $F: \mathbb{R} \times[0,1] \rightarrow \mathbb{R}$ is given by

$$
F(u, v)=u h(v)+\frac{v-h(v)}{2^{n}-1},
$$

and $\sigma: N \rightarrow N$ is a permutation such that $x_{\sigma(1)} \leq \cdots \leq$ $x_{\sigma(n)}$.

If $h(x)=k x, k \in[0,1]$, then

$$
\mathfrak{I}_{\mu}^{F}(\mathbf{x})=k \mathbf{C h}_{\mu}(\mathbf{x})+(1-k) \mathrm{OWA}_{k, n}(\mathbf{x}),
$$

where OWA operator $\mathrm{OWA}_{k, n}:[0,1]^{n} \rightarrow[0,1]$ is given by

$$
\mathrm{OWA}_{k, n}(\mathbf{x})=\sum_{i=1}^{n} \frac{2^{n-i}}{2^{n}-1} x_{\sigma(i)}
$$

Note that for any fuzzy measure $\mu \in \mathfrak{M}_{(2)}$, the values of related Möbius transform $\mathcal{M}: 2^{\{1,2\}} \rightarrow R$ satisfies $\mathcal{M}(\{1\}), \mathcal{M}(\{2\}) \in[0,1]$ and $\mathcal{M}(\{1,2\})=\mu(\{1,2\})-$ $\mu(\{1\})-\mu(\{2\}) \in[-1,1]$, and thus for $n=2$ only values of $F$ on $[-1,1] \times[0,1]$ matter when defining the function $\mathfrak{I}_{\mu}^{F}$. In this case the fuzzy measure $\mu$ is completely described by $(a, b) \in[0,1]^{2}$, where $a=\mu(\{1\})$ and $b=\mu(\{2\})$. We will write $\mu \sim(a, b)$ and $\mathfrak{I}_{\mu}^{F}=\mathfrak{I}_{(a, b)}^{F}$.

Example 4.1: Consider $n=2$ and $h:[0,1] \rightarrow\left[-\frac{1}{2}, 1\right]$ given by $h(x)=-\frac{x}{2}$. Then the corresponding function $F$ is given by $F(u, v)=-\frac{u v}{2}+\frac{v}{2}$, and related $\mathfrak{I}_{(a, b)}^{F}:[0,1]^{2} \rightarrow$ $[0,1]$ is given by

$$
\begin{aligned}
& \mathfrak{I}_{(a, b)}^{F}(x, y) \\
& =-\frac{a x}{2}+\frac{x}{2}-\frac{b y}{2}+\frac{y}{2}-(1-a-b) \frac{x \wedge y}{2}+\frac{x \wedge y}{2} \\
& =\left\{\begin{array}{cl}
\frac{(1+b) x+(1-b) y}{2} & \text { if } x \leq y, \\
\frac{(1-a) x+(1+a) y}{2} & \text { otherwise } \\
= & \mathbf{C h}_{\left(\frac{1-a}{2}, \frac{1-b}{2}\right)}
\end{array}\right.
\end{aligned}
$$

where $x \wedge y=\min \{x, y\}$.

Example 4.2: For $n=2$ and $h(x)=k x, k \in\left[-\frac{1}{2}, 1\right]$, denote the corresponding product-generalizing function $F_{k}$, i.e.,

$$
F_{k}(u, v)=k u v+(1-k) \frac{v}{3}
$$

For any $k \in\left[-\frac{1}{2}, 1\right]$ we have the unique convex representation

$$
k=\lambda \cdot\left(-\frac{1}{2}\right)+(1-\lambda) \cdot 1=1-\frac{3}{2} \lambda, \quad \lambda \in[0,1],
$$

i.e., $\lambda=\frac{2}{3}(1-k)$. Then

$$
h_{k}=\lambda h_{-\frac{1}{2}}+(1-\lambda) h_{1} \quad \text { and } \quad F_{k}=\lambda F_{-\frac{1}{2}}+(1-\lambda) F_{1} .
$$


Consequently, for any fuzzy measure $\mu: 2^{\{1,2\}} \rightarrow[0,1], \mu \sim$ $(a, b)$, we have

$$
\begin{aligned}
\mathfrak{I}_{\mu}^{F_{k}} & =\lambda \mathfrak{I}_{\mu}^{F_{-\frac{1}{2}}}+(1-\lambda) \mathfrak{I}_{\mu}^{F_{1}} \\
& =\lambda \mathbf{C h}_{\left(\frac{1-a}{2}, \frac{1-b}{2}\right)}+(1-\lambda) \mathbf{C h}_{(a, b)} \\
& =\mathbf{C h}_{\left(\frac{\lambda+2 a-3 \lambda a}{2}, \frac{\lambda+2 b-3 \lambda b}{2}\right)} \\
& =\mathbf{C h}_{\left(k a+\frac{1-k}{3}, k b+\frac{1-k}{3}\right)} .
\end{aligned}
$$

Example 4.3: For $n=2$, let $h(x)=\min \left\{x, \frac{1-x}{2}\right\}$. Then $F$ given by $F(u, v)=u h(v)+\frac{v-h(v)}{3}$ belongs to $\mathcal{F}_{2, \text { id }}$, and $\mathfrak{I}_{(a, b)}^{F}(x, y)$

$$
= \begin{cases}\mathbf{C h}_{(a, b)}(x, y) & \text { if }(x, y) \in\left[0, \frac{1}{3}\right]^{2}, \\ \mathbf{C h}_{\left(\frac{1-a}{2}, \frac{1-b}{2}\right)}(x, y) & \text { if }(x, y) \in\left[\frac{1}{3}, 1\right]^{2}, \\ (1-b)\left(x+\frac{y}{2}\right)+\frac{b}{2}-\frac{1}{6} & \text { if }(x, y) \in\left[0, \frac{1}{3}\right] \times\left[\frac{1}{3}, 1\right], \\ (1-a)\left(\frac{x}{2}+y\right)+\frac{a}{2}-\frac{1}{6} & \text { if }(x, y) \in\left[\frac{1}{3}, 1\right] \times\left[0, \frac{1}{3}\right] .\end{cases}
$$

Observe that $\mathfrak{I}_{(a, b)}^{F}$ can be represented as a Choquet integral based on level dependent fuzzy measure $\mu: 2^{\{1,2\}} \times[0,1] \rightarrow$ $[0,1]$ given by

$$
\begin{aligned}
& \mu(\{1\}, t)=\left\{\begin{array}{ll}
a & \text { if } t \leq \frac{1}{3}, \\
\frac{1-a}{2} & \text { else }
\end{array},\right. \\
& \mu(\{2\}, t)= \begin{cases}b & \text { if } t \leq \frac{1}{3}, \\
\frac{1-b}{2} & \text { else }\end{cases}
\end{aligned}
$$

For more details on fuzzy dependent measures see [11]. From another point of view, $\mathfrak{I}_{(a, b)}^{F}$ can be seen as an ordinal sum of the Choquet integrals $\mathbf{C h}_{(a, b)}$ on $\left[0, \frac{1}{3}\right]^{2}$ and $\mathbf{C h}_{\left(\frac{1-a}{2}, \frac{1-b}{2}\right)}$ on $\left[\frac{1}{3}, 1\right]^{2}$, for more details see [12].

Remark 4.6: Note that if $\mu$ is a symmetric fuzzy measure, i.e., if $\mu(A)$ depends on the cardinality of the set $A$ only, then $\mathbf{C h}_{\mu}$ is an OWA operator [13]. Considering our construction, obviously $\mathfrak{I}_{\mu}^{F}$ is symmetric whenever $\mu$ is symmetric. Hence, if $F \in \mathcal{F}_{n \text {,id }}$ and $\mu$ is symmetric, aggregation function $\mathfrak{I}_{\mu}^{F}$ can be seen as a generalized OWA operator.

So far, all results in this section were related to the identity diagonal section id. Using the same arguments, the next characterization results for a general diagonal section $\delta \in \mathcal{A}_{(1)}$ can be obtained, and thus we omit their proofs.

Theorem 4.7: Let $\delta \in \mathcal{A}_{(1)}$ and $n \geq 2$. Then the following are equivalent.

(i) $F \in \mathcal{F}_{n, \delta}$.

(ii) There is a function $h:[0,1] \rightarrow \mathbb{R}$, such that for any $x, y \in[0,1], x<y$, it holds

$$
-\frac{1}{2^{n}-2}(\delta(y)-\delta(x)) \leq h(y)-h(x) \leq \delta(y)-\delta(x),
$$

and, for all $(u, v) \in \mathcal{R}_{n} \times[0,1]$ it holds

$$
F(u, v)=u h(v)+\frac{\delta(v)-h(v)}{2^{n}-1} .
$$

The next corollary is obvious.

Corollary 4.8: The set $\mathcal{F}_{\delta}$ is a singleton, $\mathcal{F}_{\delta}=$ $\{F \mid F(u, v)=u \delta(v)\}$.
Note that due to (11), if $\delta$ is continuous (Lipschitz) on some interval $I$, also function $h$ should be continuous (Lipschitz) on $I$, but not vice-versa.

Example 4.4: Consider the greatest diagonal section $\delta^{*} \in$ $\mathcal{A}_{(1)}$

$$
\delta^{*}(x)=\left\{\begin{array}{ll}
0 & \text { if } x=0 \\
1 & \text { otherwise }
\end{array}=\mathbf{1}_{] 0,1]}(x) .\right.
$$

Then, applying Theorem 4.7, the function $F$ given by (12) belongs to $\mathcal{F}_{n, \delta^{*}}$ if and only if $h=k \cdot \mathbf{1}_{] 0,1]}$, where $k \in$ $\left[-\frac{1}{2^{n}-2}, 1\right]$. Then for any fuzzy measure $\mu \in \mathfrak{M}_{(n)}$, we have

$$
\mathfrak{I}_{\mu}^{F}(\mathbf{x})=k \cdot \mu(\operatorname{supp} \mathbf{x})+(1-k) \frac{2^{|\operatorname{supp} \mathbf{x}|}-1}{2^{n}-1},
$$

where supp $\mathbf{x}=\left\{i \in N \mid x_{i}>0\right\}$ is the support of $x$, and $|\operatorname{supp} x|$ is its cardinality. Note that, if

- $k=1$, then $\mathfrak{I}_{\mu}^{F}(\mathbf{x})=\mu(\operatorname{supp} \mathbf{x})$,

- $k=-\frac{1}{2^{n}-2}$, then $\mathfrak{I}_{\mu}^{F}=\frac{1}{2^{n}-2}\left(2^{|\operatorname{supp} \mathbf{x}|}-1-\mu(\operatorname{supp} \mathbf{x})\right)$,

- $k=0$, then $\mathfrak{I}_{\mu}^{F}(\mathbf{x})=\frac{2^{|\operatorname{supp} \mathbf{x}|}-1}{2^{n}-1}$ for any fuzzy measure $\mu \in \mathfrak{M}_{(n)}$ and the function $h$ is continuous (though $\delta^{*}$ is not).

Note that though for any fuzzy measure $\mu \in \mathfrak{M}_{(n)}$ and $F \in$ $\mathcal{F}_{n, \delta}$ the related function $\mathfrak{I}_{\mu}^{F}:[0,1]^{n} \rightarrow[0,1]$ is an aggregation function, in general, it need not extend the pseudo-boolean function $b\left(\mathbf{1}_{E}\right)=\mu(E), E \in 2^{N}$. In fact, for $F$ given by (12) we have

$$
\mathfrak{I}_{\mu}^{F}\left(\mathbf{1}_{E}\right)=h(1) \mu(E)+\frac{2^{|E|}-1}{2^{n}-1}(1-h(1)),
$$

where $h(0)=0$ is considered. Evidently, only if $h(1)=1$ (maximal possible value for $h$ ), then $\mathfrak{I}_{\mu}^{F}\left(\mathbf{1}_{E}\right)=\mu(E)$. For the minimal possible value $h(1)=-\frac{1}{2^{n}-1}$, we have

$$
\mathfrak{I}_{\mu}^{F}\left(\mathbf{1}_{E}\right)=\frac{2^{|E|}-1-\mu(E)}{2^{n}-2}=\mu_{n}(E),
$$

compare Example 3.3.

\section{CONCLUding REMARKS}

We have characterized functions $F$ leading, for some fixed diagonal section $\delta \in \mathcal{A}_{(1)}$, to an aggregation function $\mathfrak{I}_{\mu}^{F}$ with $\delta_{\mathfrak{I}_{\mu}^{F}}=\delta$, independently of fuzzy measure $\mu$. It is obvious that, for any $\delta \in \mathcal{A}_{(1)}$, the class $\mathcal{F}_{n, \delta}$ is contained in $\mathcal{F}_{n}$, i.e., $\bigcup_{\delta \in \mathcal{A}_{(1)}} \mathcal{F}_{n, \delta} \subset \mathcal{F}_{n}$. A natural open problem arises: Are there some functions $F: \mathbb{R} \times[0,1] \rightarrow \mathbb{R}$ such that, for a fixed $n \geq 2$, the function $\mathfrak{I}_{\mu}^{F}:[0,1]^{n} \rightarrow[0,1]$ is an aggregation function, for any fuzzy measure $\mu \in \mathfrak{M}_{(n)}$, but with different diagonal sections for some pair $\mu_{1} \neq \mu_{2}$ of fuzzy measures? In another words, is the system $\bigcup_{\delta \in \mathcal{A}_{(1)}} \mathcal{F}_{n, \delta}$ a proper subset of $\mathcal{F}_{n}$ ? The next example gives a positive answer to this question.

Example 5.1: Define $F: \mathbb{R} \times[0,1] \rightarrow \mathbb{R}$ by

$$
F(u, v)=\left\{\begin{array}{ll}
\frac{v}{3} & \text { if } u>-1 \\
\frac{2 v^{2}-v}{3} & \text { if } u \leq-1
\end{array} .\right.
$$

Let $n=2$. For any fuzzy measure $\mu \in \mathfrak{M}_{(2)}$ different from the greatest fuzzy measure $\mu^{*}$ given by $m^{*}(\emptyset)=0$ and $m^{*}(A)=$ 
1 otherwise, the range of the related Möbius transform $\mathcal{M}$ is contained in ] $-1,1]$, and thus

$$
\mathfrak{I}_{\mu}^{F}(x, y)=\frac{2 \min \{x, y\}+\max \{x, y\}}{3}
$$

is an OWA operator (which is idempotent, i.e., with diagonal $\delta=\mathrm{id})$. On the other hand

$$
\begin{aligned}
\mathfrak{I}_{\mu^{*}}^{F}(x, y) & =\frac{x}{3}+\frac{y}{3}+\frac{2(\min \{x, y\})^{2}-\min \{x, y\}}{3} \\
& =\frac{2(\min \{x, y\})^{2}+\max \{x, y\}}{3} .
\end{aligned}
$$

Evidently, $\mathfrak{I}_{\mu^{*}}^{F}$ is an aggregation function, but the related diagonal $\delta \neq \mathrm{id}$,

$$
\delta(x)=\frac{2 x^{2}+x}{3}
$$

The above example shows that a complete description of classes $\mathcal{F}_{n}, n \geq 2$, is still missing and it is an interesting topic for the further research.

Note that recently some alternative approaches to generalization of the Choquet integral related to the Möbius transform were introduced and studied. For example, in [14], the formula (3) was modified replacing the minimum operator. In particular, considering the product instead of minimum, the Owen (multilinear) extension of fuzzy measures is obtained [15]. A related approach introducing and studying the inclusionexclusion integrals was given in [16], see also [17]. In this case, a Möbius-like transformation of the interaction operator (generalizing both the minimum and the product over a fixed set of coordinates) is considered. As an interesting open problem one can study the generalization of the inclusionexclusion integrals replacing the product considered there by some other appropriate function.

\section{ACKNOWLEDGMENTS}

This work was supported by the Slovak Research and Development Agency under the contract no. APVV-17-0066, grant VEGA 1/0682/16, grant VEGA 1/0614/18, project of Grant Agency of the Czech Republic (GAČR) no. 18-06915S and TIN2016-77356-P(AEI/FEDER,UE).

\section{REFERENCES}

[1] G. Beliakov, A. Pradera, T. Calvo, Aggregation Functions: A Guide for Practitioners, Springer, Berlin, 2007.

[2] G. Choquet, "Theory of capacities," Annales de l'Institut Fourier, vol. 5, pp. 131-295, 1953-1954.

[3] T. Murofushi, M. Sugeno, M. Machida, "Non-monotonic fuzzy measures and the Choquet integral," Fuzzy Sets and Systems, vol. 64, no.1, pp. 7386, 1994.

[4] G. Beliakov, H. Bustince, T. Calvo, A practical guide to averaging functions Series: Studies in Fuzziness and Soft Computing, Springer, Heidelberg, 2016.

[5] G. Lucca, J. Sanz, G. Dimuro, B. Bedregal, H. Bustince, R. Mesiar, " $C_{F}$-integrals: A new family of pre-aggregation functions with application to fuzzy rule-based classification systems," Information Sciences, vol. 435, pp. 94-110, 2018

[6] G. Lucca, J. Sanz, G. Dimuro, B. Bedregal, M. J. Asiain, M. Elkano, H. Bustince, "CC-integrals: Choquet-like Copula-based aggregation functions and its application in fuzzy rule-based classification systems," Knowledge-Based Systems, vol. 119, pp. 32-43, 2017.

[7] M. Sugeno, "Theory of fuzzy integrals and its applications," Ph.D. thesis, Tokyo Institute of Technology, 1974.
[8] L. Lovász, "Submodular functions and convexity," in Mathematical Programming, 11th International Symposium, Bonn, 1982, pp. 235-257.

[9] G. Lucca, J. Sanz, G. Dimuro, B. Bedregal, R. Mesiar, A. Kolesárová, H. Bustince, "Pre-aggregation Functions: Construction and an Application," IEEE Transactions on Fuzzy Systems, vol. 24, no.2, pp. 260-272, 2016.

[10] J. Aczél, Lectures on Functional Equations and Their Applications, Academic Press, 1966.

[11] S. Greco, B. Matarazzo, S. Giove, "The Choquet integral with respect to a level dependent capacity," Fuzzy Sets and Systems, vol. 175, pp. 1-35, 2011.

[12] R. Mesiar, P. Smrek, "Choquet-like integrals with respect to leveldependent capacities and $\varphi$-ordinal sums of aggregation functions," Kybernetika, vol. 51, pp. 420-432, 2015.

[13] R.R. Yager, "On ordered weighted averaging aggregation operators in multicriteria decisionmaking," IEEE Trans. Syst. Man Cybern., vol. 18, no. 1 , pp. $183-190,1988$.

[14] A. Kolesárová, A. Stupňanová, J. Beganová, "Aggregation-based extensions of fuzzy measures," Fuzzy Sets and Systems, vol. 194, pp. 1-14, 2012.

[15] G. Owen, "Multilinear extensions of games," in A. E. Roth (Ed.), The Shapley Value. Essays in Honour of Lloyd S. Shapley, Cambridge University Press, pp. 139-151, 1988.

[16] A. Honda, Y. Okazaki, "Theory of inclusion-exclusion integral," Information Sciences, vol. 376, pp. 136-147, 2017.

[17] A. Honda, Y. Okazaki, "Generalization of inclusion-exclusion integral for nondiscrete monotone measure space," Fuzzy Sets and Systems, vol. 355 , pp. 42-58, 2019 